\title{
Estimation of Local Data-Insufficiency in Motion-Corrected Helical CT
}

\author{
Tao Sun, Student Member, IEEE, Rolf Clackdoyle, Jung-Ha Kim, Member, IEEE, \\ Roger Fulton, Senior Member, IEEE, and Johan Nuyts, Senior Member, IEEE
}

\begin{abstract}
Previously we have proposed a reconstruction algorithm which corrects for known rigid motion in helical CT. This paper describes a method to determine data-insufficiency of helical CT data affected by rigid object motion. We propose a local measure that quantifies the degree to which Tuy's completeness condition is violated in each voxel. This measure identifies regions for which artifact-free reconstruction is not assured. For every voxel, a local data-insufficiency measure is computed. We call the resulting image the Tuy map. Its values range from 0 to 1 , where high values indicate data-insufficiency. As shown by classic theory, exact reconstruction is not possible, where the Tuy map contains high values. The predictions based on this Tuy map were verified with simulated helical-CT data, where the object moved during the scan and the motion was correctly taken into account during reconstruction. We also analyzed the reconstruction from an actual motion-corrected CT-scan of a moving phantom. For motion-free helical scans, the Tuy map was close to zero everywhere and the reconstructions were artifact-free. Rigid motion induces an effective source trajectory (with respect to a stationary patient) which, combined with axial or even transaxial truncation, can cause incomplete sampling. In simulations with severe motion or transaxial detector truncation, the Tuy map contained high values and the reconstructions suffered from artifacts. In the phantom scan, the combination of a high pitch and severe motion created artifacts which the Tuy map successfully indicated. In all cases, the Tuy map indicated all regions with incomplete sampling which were prone to reconstruction artifacts. However, we also contrived a special case, where exact reconstruction was not assured although the local Tuy condition was satisfied. The proposed method provides a useful measure of data-incompleteness, which can be used to verify the validity of motion-corrected helical CT scans. The method is general and could also be useful for other tomographic problems for which no exact data sufficiency measures are available.
\end{abstract}

Manuscript received February 10, 2017; revised May 2, 2017; accepted May 24, 2017. Date of publication May 31, 2017; date of current version June 29, 2017. This work was supported in part by the Flemish Agency for Innovation by Science and Technology under Grant IWT 130065, in part by the IMIR PF-Project of KU Leuven, and in part by National Health and Medical Research Council Project under Grant 632677. (Corresponding author: Tao Sun.)

T. Sun and J. Nuyts are with the Department of Imaging and Pathology, KU Leuven (University of Leuven), 3000 Leuven, Belgium, and also with the Nuclear Medicine and Molecular Imaging, Medical Imaging Research Center, 3000 Leuven, Belgium (e-mail: colddiesun@gmail.com).

R. Clackdoyle is with TIMC-IMAG Laboratory, Université Grenoble Alpes, 38707 La Tronche, France.

J.-H. Kim was with the Faculty of Health Sciences, University of Sydney, Sydney, NSW 2006, Australia. She is now with the Faculty of Medicine, University of Sydney, Sydney, NSW 2006, Australia.

R. Fulton is with the Faculty of Health Sciences, University of Sydney, Sydney, NSW 2006, Australia, and also with the Department of Medical Physics, Westmead Hospital, Sydney, NSW 2145, Australia.

Color versions of one or more of the figures in this paper are available online at http://ieeexplore.ieee.org.

Digital Object Identifier 10.1109/TRPMS.2017.2710237
Index Terms-Data-insufficiency, helical CT, motion correction.

\section{INTRODUCTION}

$\mathbf{M}$ OTION is one of the main causes of resolution loss and artifacts in head CT imaging. We have previously developed a reconstruction method which corrects for known rigid motion in helical CT scanning of the head [1]. The motion can be measured using an external, marker-based motion tracking device [2], [3] or it can be derived from the CT-data themselves [4]. The motion correction is achieved by incorporating the known rigid motion into the system matrix of an iterative reconstruction algorithm.

Our simulation and phantom experiments indicate that with appropriate motion correction, the reconstructed images are generally artifact-free. However, in some cases the motioncorrected images do have artifacts, even if the exact motion model is used during the reconstruction [3]. We have provided evidence that this is due to data-insufficiency-i.e., in some cases the combination of head motion and scanner rotation limits projection sampling to the extent that artifact-free reconstruction is no longer assured at all points within the object. This is purely due to a limitation of the data, rather than the motion compensation scheme.

Patient motion during the scan effectively changes the acquisition source trajectory: in a coordinate system attached to the object, the CT detector and source can follow an arbitrary trajectory, created by the combination of the rigid patient motion with the ideal helical trajectory. The intended helical trajectory provides sufficient data for "exact" reconstruction, but this is not guaranteed for the modified trajectory created by the additional patient motion. Exact here means that in the ideal continuous case, the trajectory produces data which are sufficient for accurate and stable reconstruction. Experience shows that when theoretical analysis predicts exact reconstruction, accurate reconstructions are obtained in practice with analytical and/or iterative algorithms, in spite of the unavoidable discretization (to model the finite number of measurements) and in spite of deviations from the ideal model, such as noise, scatter, etc. provided these are not excessive.

Consequently, a method is needed to assess the inherent data sufficiency of the motion-corrected CT trajectory that is independent of the choice of reconstruction algorithm. This method would give a warning when the CT-scan does not provide sufficient data without having to reconstruct the images. It is important to identify regions of data-insufficiency, because 
artifacts may not always be recognized as such and may adversely affect the diagnosis. In addition, such a method would provide a tool to study how the combination of typical patient motions with different CT-scan parameters (rotation speed, pitch, axial detector size, etc.) affect data sufficiency. This tool would facilitate the definition of CT acquisition protocols which are robust to patient motion [3]. Finally, it would also be a useful tool for quality control: if artifacts are observed which cannot be attributed to data-insufficiency, then the imaging procedure has a problem which must be identified and corrected.

However, the assessment of data sufficiency (or insufficiency) in motion-corrected helical CT faces two problems. First, for cone-beam CT with truncation and an arbitrary source trajectory, there is currently no complete theory that can tell whether a particular voxel can be reconstructed exactly or not [5]. Second, even with untruncated data, the existing analytical methods only provide a binary answer, whereas for practical use, it would be preferable to obtain a degree of datainsufficiency. These two issues are discussed in the next two paragraphs.

Orlov [6] derived a data sufficiency condition for untruncated parallel-beam tomography. Tuy [7] extended this to a data sufficiency condition for untruncated cone-beam tomography. Metzler et al. [8] have identified a geometrical connection between the Orlov and Tuy sampling criteria. In that work they proposed an algorithm to compute the largest completely sampled region for arbitrary orbits in cone-beam and parallel-beam tomography. The algorithm assumes untruncated projections and verifies for each voxel if it belongs to the completely sampled region or not. Unfortunately, in helical CT, all projections are truncated due to the limited axial detector size, and transaxial truncation exists in a region-of-interest (ROI) scan. For helical trajectories with axial truncation only, data sufficiency theory has been established [9], [10]. However, there is currently no data sufficiency condition for truncated projections that is general enough to deal with an arbitrary trajectory, such as the effective trajectory caused by rigid patient motion. By inverting Tuy's condition, Finch [11] established a local data-insufficiency condition that applies to untruncated and truncated projections. This will enable us at least to identify locations, where the arbitrary source trajectory definitely created data-insufficiency, but unfortunately, it may fail to identify all such locations. One aim of this contribution is to verify how useful this local Tuy condition is as a predictor of artifacts and in which cases it may fail.

In addition, as previously mentioned, where analytical data sufficiency conditions can be applied, they provide a binary answer: at each voxel location, exact reconstruction is either possible or not. In practice, it is preferable to have a method that provides a degree of insufficiency. As an example, Tuy's condition implies that with circular conebeam tomography, exact reconstruction is only possible for the central plane. Experience shows that in practice, excellent reconstructions are also obtained for other nearby planes; the so-called cone-beam artifacts only become problematic for planes far from the central one. This agrees with the intuitive understanding that Tuy's condition is "more violated" in planes more distant from the central plane. Practical methods to determine the degree of sampling insufficiency have been proposed for various geometries. Metzler et al. [12] proposed a metric of measuring sampling sufficiency for a pinhole single photon emission computed tomography (SPECT) system. Clackdoyle and Noo [13] provided a quantitative prediction about data sufficiency in cone-beam reconstruction for a hypothetical pinhole SPECT system for breast imaging. Liu et al. [14] proposed a completeness map for several candidate cardiac CT system designs.

Also analytical algorithms could be used to study datainsufficiency. Analytical algorithms similar to filtered backprojection make strong assumptions about the trajectory of the $\mathrm{X}$-ray source. In contrast, analytical reconstruction algorithms based on chords (Pi-lines) can reconstruct images from scans along irregular trajectories [15]. Unfortunately, the requirements of these chord-based methods are sufficient but not necessary for exact reconstruction, e.g., for particular voxels, severe motion could break up the trajectory into disjoint parts, such that these voxels are not on a chord, while exact reconstruction would still be possible [16]. Yu et al. [17] developed a backprojection and filtering algorithm that is capable of reconstructing also voxels not lying on a chord. However, using such methods to verify if a particular point can be reconstructed or not requires a rather extensive analysis. In addition, the method would then produce a binary answer, not a continuous value assessing the degree to which Tuy's condition is violated.

In this paper, we propose a measure to quantify the degree to which Tuy's condition is violated in every voxel for a CT-system with an arbitrary trajectory. The method is general, but our focus is on motion-corrected helical CT using a clinical system. The method is specific: voxels found to violate the local Tuy condition definitely pose a reconstruction problem [11] but its sensitivity is not perfect (some regions suffering from data-insufficiency may not be detected). The method is independent of the reconstruction algorithm, as it identifies voxels for which the data are insufficient based on theory. We evaluate our method with an iterative reconstruction algorithm. We verify that artifacts appear, where predicted by the method. We also study cases, where the method is expected to fail, i.e., where the reconstruction problem is not unique due to data-insufficiency even though the local Tuy condition is satisfied.

\section{Methods And Materials}

\section{A. Voxel-Based Tuy Map}

Tuy provided a sufficient condition for exact cone-beam reconstruction from untruncated projections: "if on every plane that intersects the object, there exists at least one cone-beam source position, then one can reconstruct the object" [7]. As explained in the Introduction, we cannot directly apply Tuy's condition to measure projection insufficiency for (motion-corrected) helical CT, due to the inevitable projection truncation.

Finch [11] proved the converse of Tuy's condition: "if there is a plane which contains no measurement lines passing through a particular voxel, then stable reconstruction is not 
possible for that voxel." This condition provides theoretical support for our incompleteness measure for arbitrary conebeam trajectories with truncated projections. Based on Finch's result, we define a voxel-based measure of data-insufficiency. We call this the local Tuy value, and refer to the corresponding image as the Tuy map.

The overall procedure to obtain the Tuy map is as follows. To approximate the continuous model, the size of the voxel is chosen "small enough," i.e., smaller than the detector pixel size. For every voxel, planes passing through the center of that voxel are considered. For every voxel, we also define a set of measured projection lines: we examine all the source positions of the CT acquisition, and for each source position on the effective trajectory, the line containing the source position and the center of the voxel is considered. If that line intersects the detector, then we consider this line as a measured projection line.

A plane through a specified voxel can be represented by its unit normal vector, and the measured projection lines through the voxel by the unit vectors in the directions of the lines. The absolute values of the dot products of the projection line vectors with the plane normal vector are computed, and the minimum is stored for each plane. The local Tuy value for the voxel is finally calculated as the maximum of the stored values across all planes. This procedure finds for each voxel the plane that most severely violates the local Tuy condition, and quantifies that violation by examining the angle between the plane and the "most parallel" projection line. If the line is in the plane (intersection angle is zero), the value is zero. The procedure is described in more detail in Fig. 1, in which step 2 involves sampling of a unit hemisphere [18].

The local Tuy values range from 0 to 1 , a higher Tuy value in a particular voxel indicates that the local Tuy condition is violated more severely within that voxel. A value of zero indicates that all planes through the voxel contain at least one measured projection line.

To clarify the meaning of the Tuy value, consider a set of 2-D parallel-beam projections acquired with an angular coverage $[0, \alpha]$, where $\alpha$ varies from 0 to $2 \pi$. It is known that the sampling is complete when $\alpha$ is in $[\pi, 2 \pi]$, while for $\alpha$ in $[0, \pi)$, the sampling is incomplete. In addition, it has often been observed that the limited angle artifacts become worse when the angular coverage $[0, \alpha]$ is further reduced. In this 2-D case, the planes considered for the Tuy condition reduce to lines (see Fig. 2). It is easy to verify that the "Tuy line" with the largest minimum dot product $|\vec{l} \cdot \vec{n}|$ makes an angle $(\pi-\alpha) / 2$ with the $x$-axis when $\alpha \in[0, \pi)$. Therefore, the Tuy value for all voxels in the field of view (FOV) equals $|\vec{l} \cdot \vec{n}|=\sin ((\pi-\alpha) / 2)$ if $\alpha \in[0, \pi)$ and zero elsewhere. Fig. 3 shows a plot of the Tuy values. For $\alpha=72^{\circ}, 144^{\circ}$, and $180^{\circ}$, sinograms of a 2-D spheres phantom have been simulated and reconstructions with maximum likelihood expectation maximization (MLEM) were made, illustrating that artifact severity increases with increasing Tuy value.

This approach has two sources of discretization and associated discretization errors. The first one is the discretization created by only considering source positions corresponding to actual CT measurements. Since a CT-scan typically contains
1. Find all projection lines $\{\vec{l}\}$ passing through the voxel

for all projection angles/views do

-apply the inverse motion to projector

-calculate the source position $O$

-calculate the position of detector corners

$C 1, C 2, C 3, C 4$

-calculate the intersection point $I$ on the detector

if $I$ is located inside detector surface then

end if

return $\vec{l}=\overrightarrow{O I}$

end for

2. Find all unit normal vectors $\{\vec{n}\}$ representing planes through the voxel, by uniformly sampling half of a unit sphere.

for all sampling points do

get the sampling point coordinate $S$

return $\vec{n}=S$

end for

3. Compute Tuy value from worst plane.

normalize the $\{\vec{l}\}$

for all $\vec{n}$ in $\{\vec{n}\}$ do

$$
\begin{aligned}
& \quad \text { return } m_{\vec{n}}=\min _{\vec{l}}|\vec{l} \cdot \vec{n}| \\
& \text { end for } \\
& \text { return localTuyvalue }=\max _{\vec{n}}\left\{m_{\vec{n}}\right\}
\end{aligned}
$$

Fig. 1. Pseudo-code representation of the voxel-based Tuy value computation.

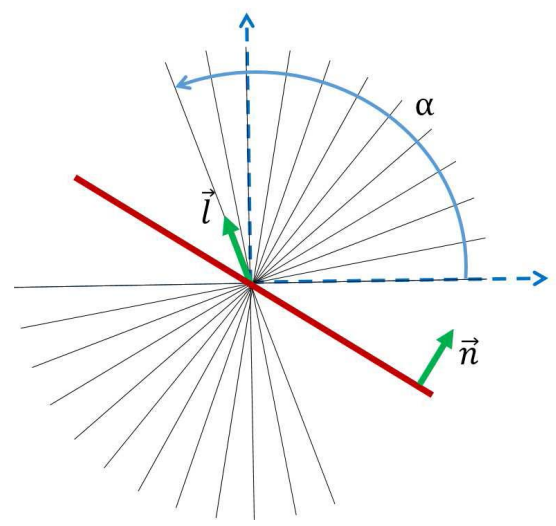

Fig. 2. Example of 2-D imaging with limited angle tomography $\alpha<\pi$. The projection lines available for a particular voxel are shown. For the plane shown in red, the dot product between its normal $\vec{n}$ and the projection line is minimum for the indicated projection $\vec{l}$. The indicated plane is the one that has the largest minimum dot product $|\vec{l} \cdot \vec{n}|$.

thousands of views per rotation, the effects of this discretization are very small, and we found that useful Tuy maps could be obtained even with a reduced sampling of a few hundred samples per rotation. The second discretization is determined by the number of samples on the unit hemisphere. This number can be chosen arbitrarily, in our experience 500 samples are sufficient to obtain useful Tuy values. The method is not affected by detector discretization (it only uses the detector boundary to verify if a projection line intersects the detector), nor by discretization of the image into voxels (the Tuy value 
TABLE I

SCANNing PARAMEters Settings

\begin{tabular}{llll}
\hline & $\begin{array}{l}\text { Configuration 1 } \\
\text { (Section III-A, III-B, III-C) }\end{array}$ & $\begin{array}{l}\text { Configuration 2 } \\
\text { (Section III-D) }\end{array}$ & $\begin{array}{l}\text { Configuration 3 } \\
\text { (Section III-E) }\end{array}$ \\
\hline Scanner model & Definition AS & Sensation 16 & Sensation 16 \\
Tube voltage $(\mathrm{kVp})$ & N/A & 120 & N/A \\
Tube current $(\mathrm{mA})$ & N/A & 150 & N/A \\
Rotation time $(\mathrm{s})$ & N/A & 0.5 & N/A \\
Pitch & 0.8 & 1.0 & 1.0 \\
Angles per rotation & 500 & 1050 & 500 \\
Collimation $(\mathrm{mm})$ & $32 \times 1.2$ & $16 \times 0.75$ & $16 \times 0.5$ \\
Flying focus & off & off & off \\
Reconstruction pixel size $\left(\mathrm{mm}^{3}\right)$ & $2.0 \times 2.0 \times 1.0$ & $1.0 \times 1.0 \times 1.0$ & $1.6 \times 1.6 \times 1.0$ \\
Dimension & $160 \times 160 \times 120$ & $512 \times 512 \times 285$ & $200 \times 200 \times 120$ \\
Reconstruction updates & 5 iterations, 20 subsets & 6 iterations, 40 subsets & 50 iterations, 20 subsets \\
\hline
\end{tabular}

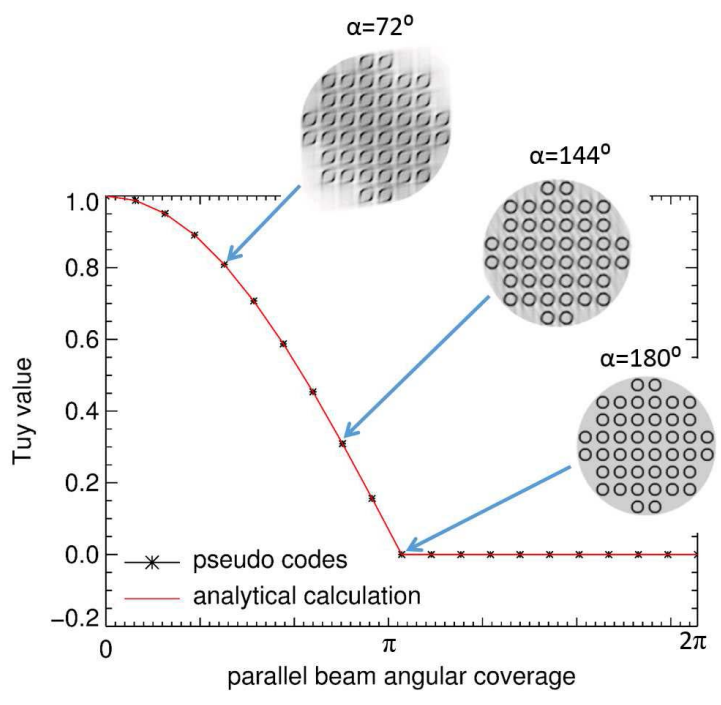

Fig. 3. Red line represents the analytical calculation of the Tuy values at one voxel for parallel-beam geometries with angular coverage $[0, \alpha]$, where $\alpha$ varies from 0 to $2 \pi$. The black star dots are the Tuy values calculated according to pseudo codes. The three images are from the corresponding 2-D reconstructions when $\alpha=72^{\circ}, 144^{\circ}$, and $180^{\circ}$, respectively.

can be computed everywhere, our choice to only compute it at voxel centers is arbitrary).

\section{B. Scanner Geometry and Simulation}

For different simulation experiments, helical scans were simulated for the geometry of the Siemens Definition AS CT scanner and Siemens Sensation 16 scanner (Siemens Medical Solutions USA, Inc., Malvern, PA, USA). In addition, a phantom scan was performed on a Siemens Sensation 16 scanner (incorporated in a positron emission tomography/computed tomography system). A sketch of the system is given in Fig. 4. The scan parameters used in different studies can be found in Table I.

In the simulations, we introduced a geometric mismatch with the forward model used by the image reconstruction, by upsampling both the object and the detector by a factor of 2 in all directions when generating the projections. We assumed uniform blank scans, monochromatic radiation and
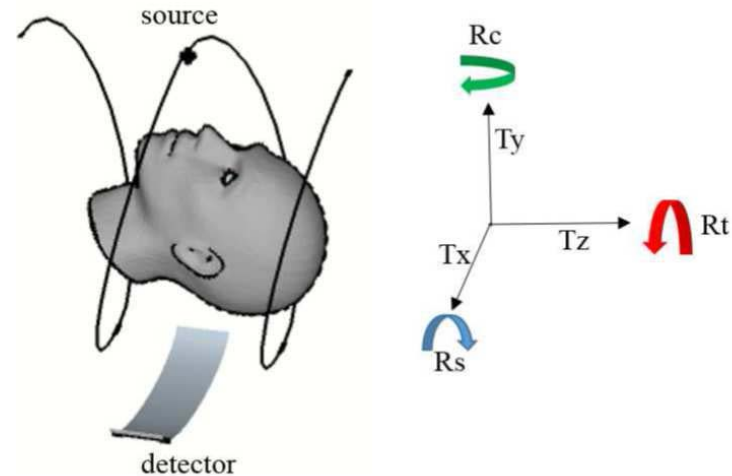

Fig. 4. X-ray source moves in a helical trajectory. The six degrees-offreedom rigid motion are shown in the given scanner coordinate system. $T_{z}$ is the translation along the $z$-axis (bed direction), $T_{x}$ is the translation along the $x$-axis, and $T_{y}$ is the translation along the $y$-axis. $R_{t}$ is the rotation around the $z$-axis, $R_{S}$ is the rotation around the $x$-axis, and $R_{C}$ is the rotation around $y$-axis.

ignored scatter in all simulations, since we did not want any confounding effects when evaluating the Tuy map.

When motion was present, it was simulated by applying the inverse motion to the CT-detector gantry for each individual view. For some simulations, the motion was based on recorded volunteer head motions, for others some (highly artificial) motion patterns were synthesized.

\section{Phantom Used in Simulations}

For this paper two software phantoms were created. The first phantom, called the multisphere phantom, consisted of a large cylinder filled with small hollow spheres. This ensured that it had sharp edges in all directions, such that creation of reconstruction artifacts was likely wherever data-insufficiency occurred. The spheres had an outer diameter of $24 \mathrm{~mm}$, an inner diameter of $20 \mathrm{~mm}$ and were placed on a rectangular grid of $32 \times 32 \times 24 \mathrm{~mm}^{3}$. The sphere shell attenuation was $0.04 \mathrm{~mm}^{-1}$ (which is similar to the attenuation of cortical bone at $70 \mathrm{keV}$ ). The background cylinder had a diameter of $256 \mathrm{~mm}$, a height of $192 \mathrm{~mm}$ and an attenuation coefficient of $0.01 \mathrm{~mm}^{-1}$. For the simulation, the phantom was discretized 


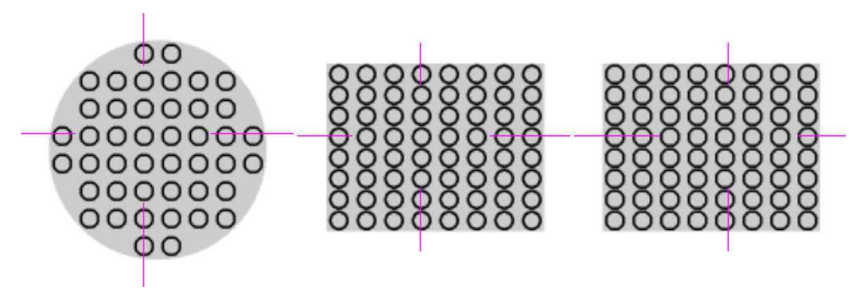

Fig. 5. Transaxial, coronal, and sagittal view of the multisphere phantom. The purple lines are crosshairs specifying the location of the other two orthogonal slices.

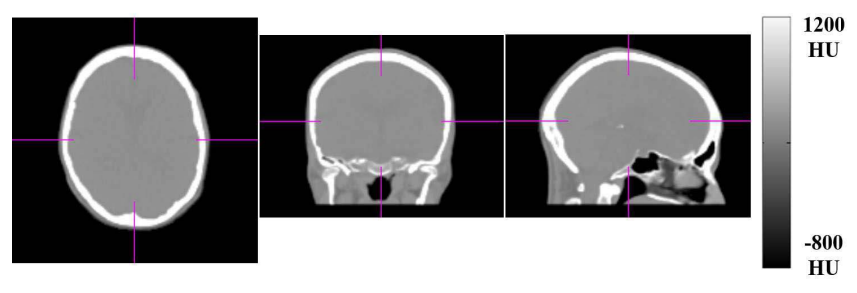

Fig. 6. Three orthogonal views of the voxelized head phantom.

using nearest neighboring interpolation in an image of $320 \times$ $320 \times 240$ voxels with a voxel size of $1 \times 1 \times 0.5 \mathrm{~mm}^{3}$.

The second phantom was a voxelized head phantom, created from the CT image of an anthropomorphic phantom by interpolating it to a grid of $320 \times 320 \times 240$ voxels with a voxel size of $1 \times 1 \times 0.5 \mathrm{~mm}^{3}$.

After the simulation of the CT scan(s) the detectors were resampled by summing $2 \times 2$ pixels for both phantoms, as mentioned above, and the images were reconstructed into a grid with voxels of $2 \times 2 \times 1 \mathrm{~mm}^{3}$ covering the same FOV as used for the simulation.

\section{Motion Recording}

For simulations, we used six degrees-of-freedom head motion data acquired from three healthy volunteers using an infrared optical tracking system (Optitrack Flex 13, Natural Point Inc, Corvallis, OR, USA). One volunteer was asked to move the head moderately, another two were asked to move their heads as much and as rapidly as possible. The head motion was recorded while the volunteers were lying on the CT-bed, but no CT scan was acquired. More details about acquiring the motion data can be found in [3], which also provides the recorded motions as supplementary data.

For the phantom scan, the experiment was similar to one described in [2]. A Hoffman brain phantom (diameter $17.5 \mathrm{~cm}$, height $20.8 \mathrm{~cm}$, filled with air) was initially supported by a wedge in an elevated position on the curved surface of the bed. Just before the scan the wedge was removed by pulling a string from outside the room. This caused the phantom to roll from side to side 5 or 6 times on the concave bed with diminishing amplitude for the remainder of the scan. The movement lasted for approximately $10 \mathrm{~s}$. Most of this motion consisted of translations and rotation in the transaxial plane. However, there was also motion out of the transaxial plane: in its original elevated position, the axis of the phantom was not aligned to the axis of the bed, and the phantom aligned itself while rolling on the bed. The CT scan was performed while the motion was recorded.

All the acquired motion data were converted to isocenter coordinates, smoothed with a second degree polynomial to reduce measurement jitter, and linearly interpolated to obtain the pose at the time of each projection acquisition, as described in [2].

\section{E. Reconstruction With Motion Correction}

In the presence of motion, the helical CT source trajectory is distorted into an effective trajectory with arbitrary shape. As discussed above, an arbitrary effective trajectory with truncation presents challenges for analytical reconstruction algorithms, which we have avoided by using an iterative reconstruction algorithm. In this paper, we used the MLEM reconstruction algorithm [19]. Actually, this is standard MLEM with an adjusted system matrix. Instead of moving the reconstruction image, rigid motion correction was done by considering a coordinate system fixed to the object and incorporating the motion (now associated to the source-detector pair) into the system matrix [4]. This corresponds to an arbitrary 3-D motion of the virtual gantry around the object being scanned. Because of the high rotation speed and the large number of views, the motion within a single view is negligible.

The ordered subsets expectation maximization (OSEM) was adopted to speed up the convergence of the iterative reconstructions [20]. The number of iterations and subsets used in all reconstructions are specified in Table I. All projectors/backprojectors were implemented using the distancedriven approach [21].

To illustrate that the Tuy map identifies fundamental datainsufficiency which poses problems to any reconstruction algorithm (which relies on the measured data only), all reconstructions were also performed with simultaneous algebraic tomographic reconstruction (SART) [22].

\section{F. Artifact Quantification}

In simulations with the multisphere phantom, the motioncorrected reconstructed image is likely to suffer from artifacts in cases of data-insufficiency, because the object has spatial frequencies in all possible directions. Thus, for this particular phantom, the occurrence and severity of reconstruction artifacts are expected to correlate well with the degree of data-insufficiency. We performed a reconstruction of the multisphere phantom without motion to produce a reference image. When motion was included, the severity of artifacts was quantified using the root mean square error (RMSE) between the two reconstructed images, and compared with the Tuy value map from Fig. 1

$$
\mathrm{RMSE}=\sqrt{\frac{\sum_{j=1}^{N}\left(x_{j}-\tilde{x}_{j}\right)^{2}}{N}}
$$

where $x_{j}$ is the value of the $j$ th voxel of the evaluated image, $\tilde{x}_{j}$ is the corresponding value of the reference image and $N$ is the total number of voxels.

The similarity metric RMSE was computed in a blockbased fashion. We divided the reconstructed images into small 
blocks, each being a $16 \times 16 \times 12$ voxels neighborhood surrounding the center of each sphere. A similar sampling approach was applied to the Tuy value map. Therefore, the final results were two arrays: one stored a metric value quantifying possible artifacts, and one stored the block-averaged Tuy value. Comparative analysis was then performed to reveal the relationship between the local Tuy value and the severity measure of the possible artifacts, in both artifact-free regions and regions affected by artifacts.

\section{G. Interior-Like Problem Simulation}

Last, we examined a case, where the Tuy map does not predict data-insufficiency in a particular region. In the 2-D interior problem, all projections are truncated transaxially on both sides. For the pixels inside the interior region all projection lines are still available, whereas for the surrounding pixels, some lines are lost due to the truncation. The local Tuy values will be zero inside the interior region and greater than zero in the surrounding region, so the local Tuy condition implies data sufficiency for the interior region. However, for interior projection data, it is known that the solution is not unique inside the interior region. The reconstructed image may suffer from a dc-shift and low frequency artifacts in the interior region [23].

To examine the effectiveness of the proposed Tuy map on a 3-D version of the interior problem, we studied the following interior-like problem. The interior region can be created by reducing the fanangle. On the other hand, a specially designed motion can also induce such an interior region, if the outer parts of the object occasionally move out of the FOV during the helical scan. The motion was designed such that the local Tuy condition was satisfied within the ROI, but not in the volume surrounding that ROI. The created missing data problem has some similarity to that of the classical 2-D interior problem. We then checked how the reconstruction of the interior region was affected by the incomplete sampling in the surrounding structures. The hypothesis was that in this situation, the interior region of the object cannot be exactly reconstructed, although the local Tuy values are very low. Note that here the simulation is not a straightforward extension of the 2-D interior problem to 3-D, because in our 3-D problem, the low Tuy value volume is only surrounded transaxially by high Tuy values, not axially.

\section{Simulations And Phantom StUdy}

The goal was to verify the effectiveness of the proposed Tuy map in various situations. A total of five studies were performed to verify the predictions of data-insufficiency in the context of helical CT.

1) A static simulation with complete sampling-the object was static during the scan and the FOV was large enough to cover the object in all directions.

2) A simulation with moderate object motion-the object was undergoing moderate motion during the scan while the FOV remained the same as in the first simulation.

3) Three simulations, two using the multisphere phantom, and one with the voxelized head phantom, with severe

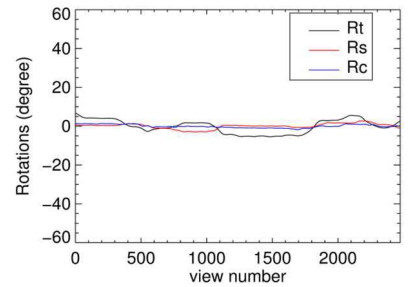

(a)

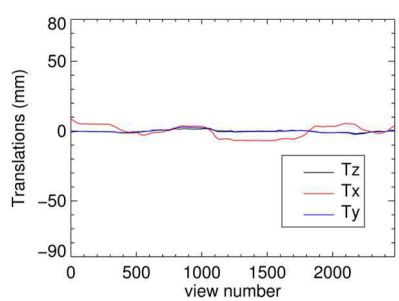

(b)
Fig. 7. Moderate rigid motion segment recorded from subject \#1. (a) Rotations. (b) Translations. All the motion recorded from volunteers can be found in the supplementary data of [2].

object motion, which was likely to create incomplete measurements.

4) A moving phantom scan - an actual helical CT scan with a moving phantom.

5) Two simulations concerning an interior-like problem. In each study, the reconstructions were compared to the Tuy maps to study the relation between the occurrence of artifacts and the local Tuy values.

\section{A. Simulation With Static Object}

A helical scan of the stationary multisphere phantom was simulated as described in Section II-B. The parameters are listed as configuration 1 in Table I. The FOV covered the whole object in the transaxial plane. The hypothesis was that this experiment would produce a Tuy map which was (almost) zero everywhere and an artifact-free reconstruction, indicating that the sampling was complete everywhere.

\section{B. Simulation With Moderate Object Motion}

A segment (Fig. 7) of the motion data from a volunteer was applied to the multisphere phantom during a helical scan simulation, as described in Section II-B. The scan parameters are listed as configuration 1 in Table I. As shown in Fig. 7, this was a moderate motion with translations up to $8 \mathrm{~mm}$ and rotations up to $7^{\circ}$. Since the motion-corrected reconstruction did not produce any artifacts, the Tuy map was expected to be (almost) zero everywhere.

\section{Three Simulations With Severe Object Motion}

1) Two Multisphere Phantom Simulations: Similar simulation experiments were performed with the same multisphere phantom, but this time with the more severe volunteer motions shown in Figs. 8 and 9. The hypothesis was that for these motions, the Tuy map would have some nonzero values due to data-insufficiency, and the reconstruction with motion correction would produce images with artifacts in those regions. The scan parameters are listed as configuration 1 in Table I.

2) Simulation With the Voxelized Head Phantom: In order to further investigate the correlation between the Tuy map and the artifacts in an image more similar to those encountered in clinical practice, we applied the motion of Fig. 8 also to the voxelized head phantom (Fig. 6). Visual assessment was performed to verify the match between the Tuy map and the motion-corrected reconstruction image. Again, we expected 


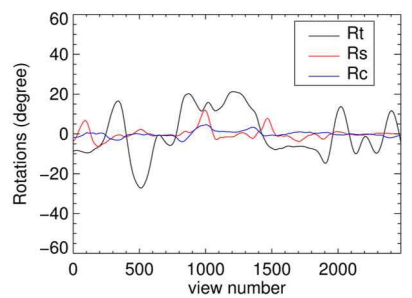

(a)

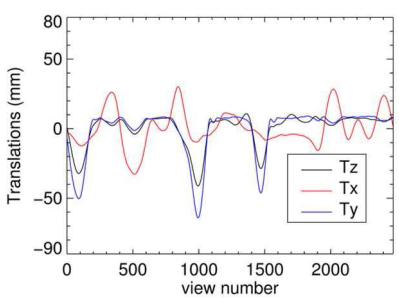

(b)
Fig. 8. Severe rigid motion segment from subject \#2. (a) Rotations. (b) Translations.

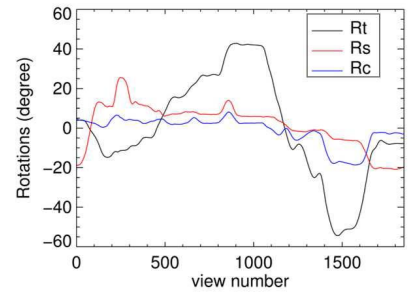

(a)

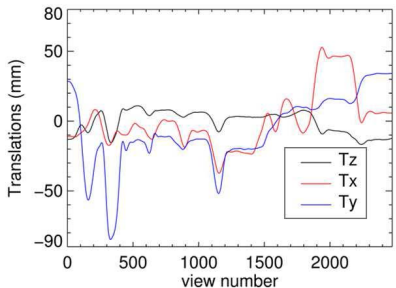

(b)
Fig. 9. Severe rigid motion segment from subject \#3. (a) Rotations. (b) Translations.

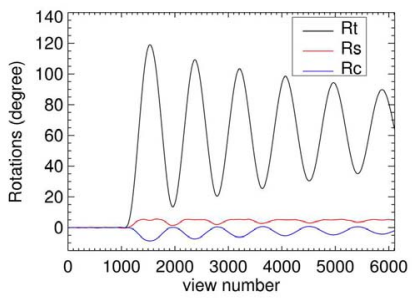

(a)

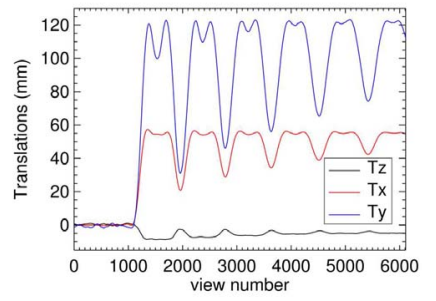

(b)
Fig. 10. Rolling motion recorded in the phantom scan. (a) Rotations. (b) Translations.

that severe artifacts would only occur in regions with high Tuy values.

\section{Moving Phantom Scan}

A 3-D Hoffman brain phantom filled with air was used. The scanning parameters are listed as configuration 2 in Table I. The motion recording setup is described in Section II-D. The rolling motion (Fig. 10), had up to $120^{\circ}$ in-plane rotation, $120 \mathrm{~mm}$ in-plane translation and $10^{\circ}$ out-of-plane rotation, $10 \mathrm{~mm}$ out-of-plane translation. To assess the datainsufficiency, the Tuy map was computed and compared to motion-corrected images.

\section{E. Two Simulations Concerning Interior-Like Problem}

The scan parameters used in this simulation are listed as configuration 3 in Table I. Unlike the previous simulations, a Siemens Sensation 16 CT scanner was simulated. The Sensation 16 has a narrower $z$-coverage than the definition AS which we chose for the other simulations. We assumed that the creation of the interior region problem would be easier for a narrow detector, since axial truncation is needed to create the outer region with high Tuy values.

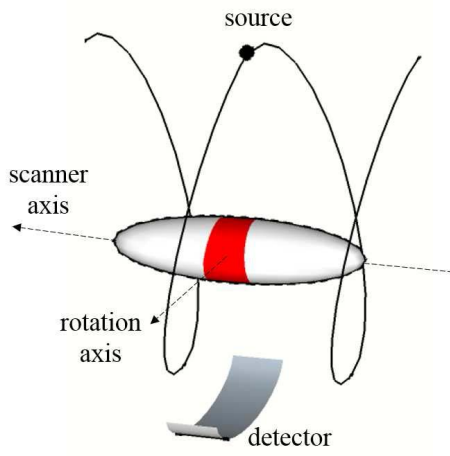

(a)

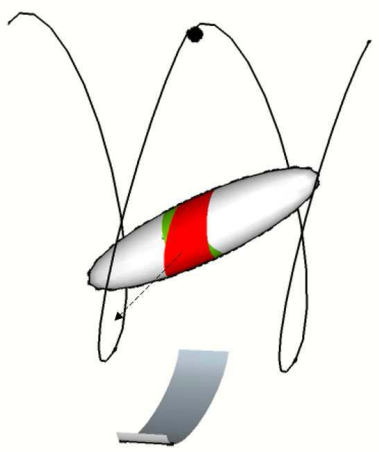

(b)
Fig. 11. Red region indicates the intersection with the FOV for the current view (a) without and (b) with rotation of the object-the green region has been moved out of the FOV (axial truncation) by the rotation.

1) Data Truncation From Reduced Fanbeam Angle: Simulations were performed with transaxial truncation. For the simulated scanner, the fanangle was reduced from $52.1^{\circ}$ to $13.7^{\circ}$. By doing this, an interior-like problem (similar to 2-D interior problem) was created as described in Section II-G. We still used the multisphere phantom shown in Fig. 5.

OSEM was performed for 50 iterations and 20 subsets to guarantee good convergence of the reconstruction. The Tuy map was computed and confirmed the creation of a 3-D interior low Tuy value region, surrounded by higher Tuy values. As discussed in Section II-G, our hypothesis was that the reconstructions would suffer from a dc-shift and/or low frequency artifacts inside the interior region, where the sampling was complete according to the Tuy map.

2) Data Truncation From Motion: As indicated in Section II-G, we designed a motion to create a pattern of truncation in the projections that would be similar to the situation of the 2-D interior problem. For each CT projection view, the object was rotated by a constant angle of $7.5^{\circ}$ about an axis located inside the transaxial plane and perpendicular to both the rotation axis of the scanner, and to the line connecting the source and the center of the detector array (Fig. 11). With such motion, there was always part of the outer object moving out of the FOV during the scan. The motion was large enough to create a pattern of motion-induced data truncation that swept over the exterior region, as the source-detector pair moved in a spiral fashion. The motion was contrived to ensure all outer parts of the object would be outside the FOV for some projection views, while the inner parts of the object always stayed within the FOV.

The motion was applied to the multisphere phantom (Fig. 5) in a simulated helical scan. The simulation and reconstruction setup were identical to the one in Section III-E1. The Tuy map was computed to confirm the creation of a 3-D interior low Tuy value region, surrounded by higher Tuy values.

\section{RESUlTS}

The results are presented in the same order as in the previous section. 


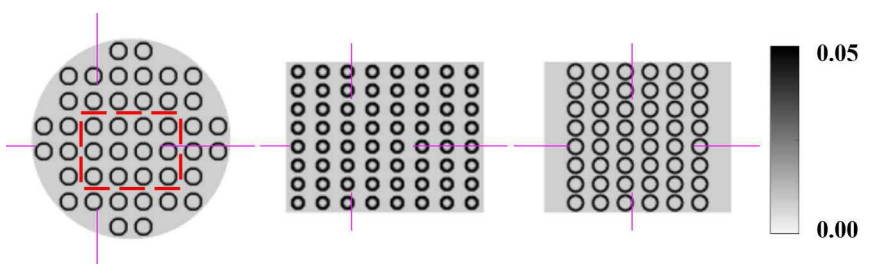

(a)
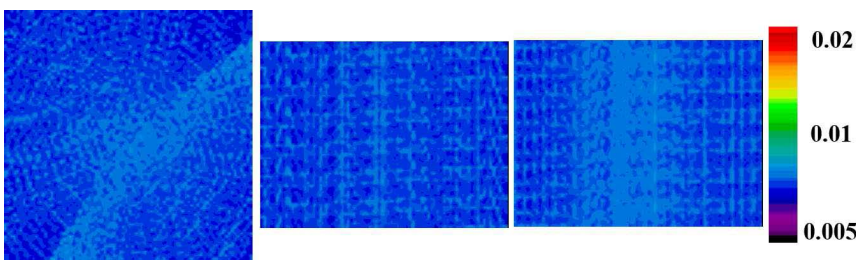

(b)

Fig. 12. Transaxial, coronal, and sagittal planes through the MLEM reconstruction from (a) motion-free CT-scan and (b) corresponding Tuy map. The red dashed square refers to Fig. 15.

\section{A. Simulation With Static Object}

Three orthogonal planes through the 3-D reconstructed images of the static multisphere phantom are shown in Fig. 12(a). These images, when compared to those of Fig. 5, suggest that the reconstruction was artifact-free everywhere, as anticipated. The same three planes through the 3-D Tuy map are shown in Fig. 12(b), revealing that all the calculated local Tuy values were between 0 and 0.01 , which we consider to be effectively zero. The small nonzero values of the Tuy map are due to the discretizations described in Section II-A. In summary, the local Tuy values were close to zero, and the reconstruction image showed no visible artifacts. These two observations are compatible with each other and with the fact that no data-insufficiency was anticipated for this simulation study.

\section{B. Simulation With Moderate Object Motion}

Three orthogonal planes through the 3-D reconstructed images of multisphere phantom undergoing moderate motion are shown in Fig. 13(a). Again, these images, when compared to those of Fig. 5, suggest that the reconstruction was artifact-free everywhere, as anticipated. The same three planes through the 3-D Tuy map are shown in Fig. 13(b), revealing that all the calculated local Tuy values were between 0 and 0.02 , which we consider to be effectively zero. In summary, the local Tuy values were close to zero, and the reconstruction image showed no visible artifacts. Hence, there was no data-insufficiency in this simulation study, as anticipated.

\section{Three Simulations With Severe Object Motion}

1) Two Multisphere Phantom Simulations: Orthogonal planes of the reconstructions with motion correction and the Tuy maps are shown in Fig. 14. Reconstruction artifacts were observed for the two sets of severe rigid motions, suggesting that the severe motions did create data-insufficiency. To facilitate comparison of the images an overlay of the Tuy values on the reconstructed image planes is added. The figure shows the

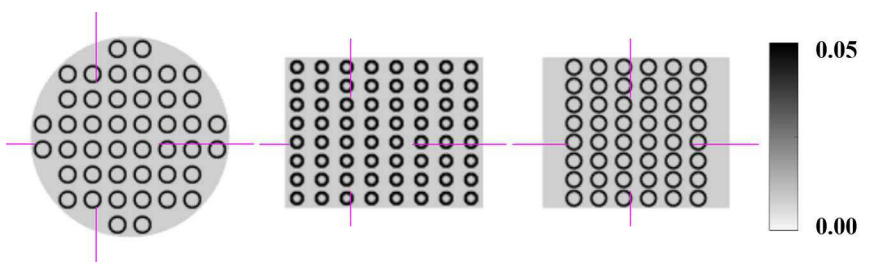

(a)
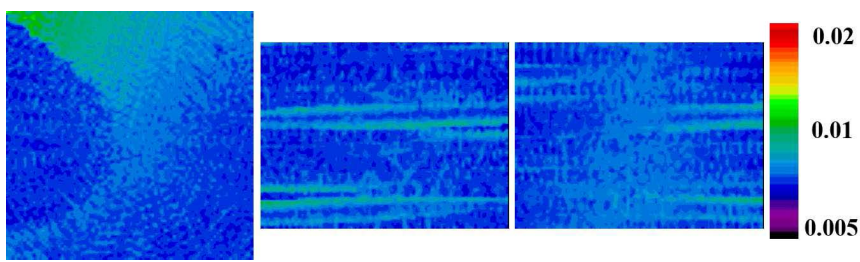

(b)

Fig. 13. Three orthogonal planes of a motion-corrected MLEM reconstruction from (a) CT-scan with moderate motion with the motion from Fig. 7 and (b) corresponding Tuy map.

planes with the highest Tuy values, and reveals good spatial matching between the occurrence of reconstruction artifacts and increased Tuy values.

As described in Section II-F, we performed a quantitative analysis for the results from the motion in Figs. 9 and 15 reveals a strong positive correlation between the severity of the artifacts and the average Tuy value for selected sampled blocks within the phantom space. From these studies, we observe that the local Tuy value was a good predictor of local artifact severity, at least for the multisphere phantom.

2) Simulation With the Voxelized Head Phantom: Fig. 16 shows the motion-corrected reconstructed images of the digital head phantom which had undergone severe motion (Fig. 8) during the scan. We observe that high local Tuy values occur at regions, where artifacts appear (red arrows in Fig. 16), but there are other regions of high Tuy values that appear artifact-free (blue arrows in Fig. 16). Fig. 17 shows that although the artifacts in SART are different from those of MLEM, the artifacts occur in the high Tuy value regions for both algorithms. The Tuy map is designed to measure datainsufficiency, but data-insufficiency does not always create artifacts in the reconstruction. The multisphere phantom has fine details with edges in all directions, making it very likely that data-insufficiency will indeed create artifacts, which was confirmed by the experiments (Figs. 14 and 15). However, typical clinical images often have large fairly uniform regions, which are less challenging for most reconstruction algorithms. In agreement with observations in [23], artifacts showed up "mainly in the vicinity of lines which are tangent to curves of discontinuity of the object and for which the value in the projections are missing." We verified that distortions were mostly seen, where the missing projection lines were nearly tangent to some of the discontinuities (a separate study was done but not shown in this simulation). The discontinuities that were perpendicular to the missing lines suffered less from artifacts. Consequently, the Tuy map indicates, where artifacts may occur, but whether they occur or not depends on the object features. 


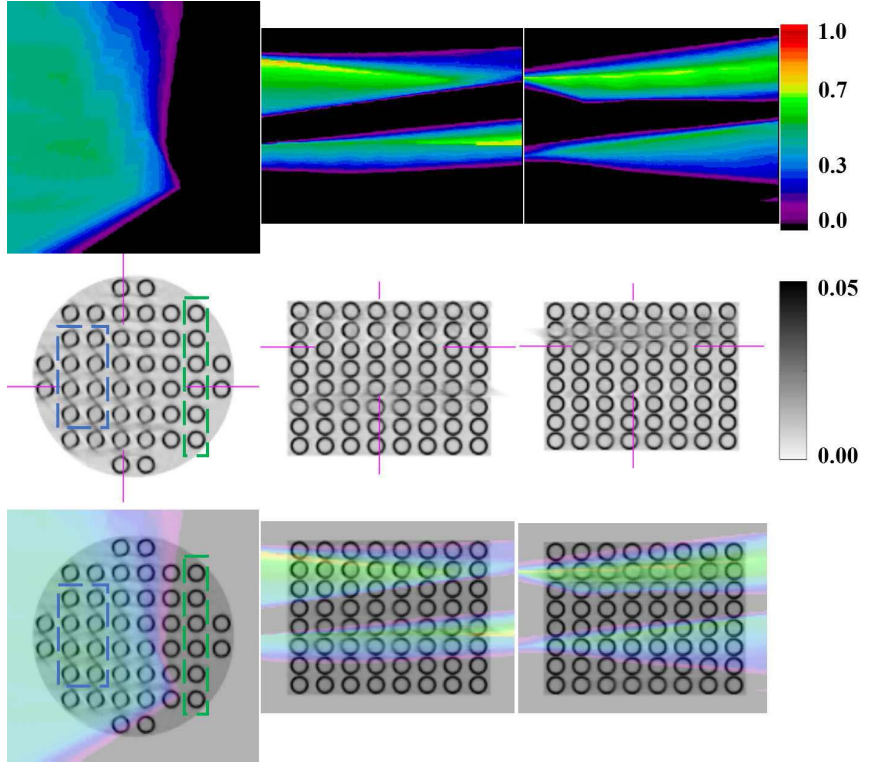

(a)
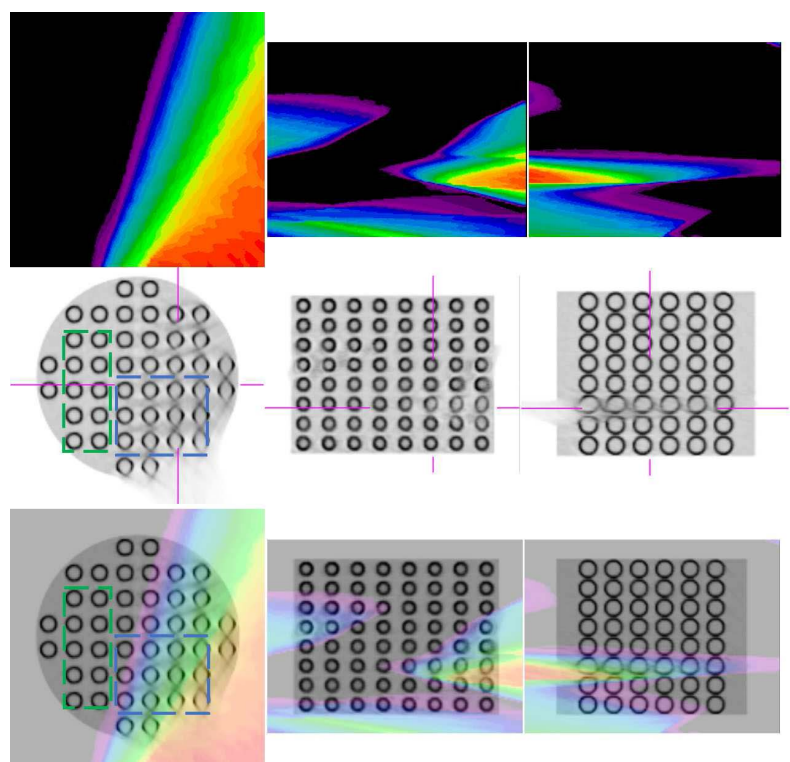

(b)

Fig. 14. Result of simulations with severe motion of the object. (a) Top: Tuy map for the scan with motion from Fig. 8; middle: MLEM reconstruction image in the same position; bottom: the image overlayed with the Tuy map. (b) Top: Tuy map for the scan with motion from Fig. 9; middle: reconstruction image in the same position; bottom: image overlayed with the Tuy map. Red indicates high Tuy values and therefore poor tomographic sampling in a particular voxel. The green (artifact-free) and blue (with artifacts) dashed squares refer to Fig. 15.

\section{Moving Phantom Scan}

Three orthogonal planes of the motion-corrected images are shown in the middle row of Fig. 18. Different from the previous studies, it is based on a real CT scan of a 3-D Hoffman phantom. The artifacts are clearly visible in all three views. The same three views through the 3-D Tuy map are shown in the top row of Fig. 18. The regions with high Tuy-values indicate the potential risk of artifacts in those regions. The overlayed image (bottom, Fig. 18) indicates that the Tuy map

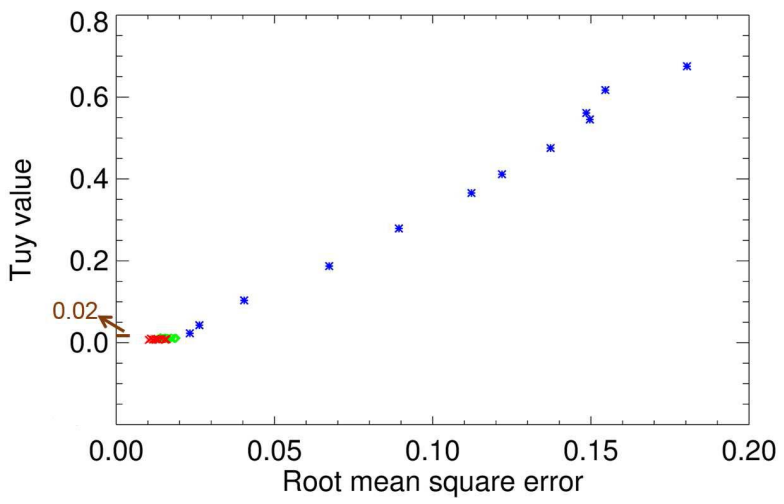

Fig. 15. Scatter plot shows the relationship between RMSE (reconstructions versus true phantom) and sampled Tuy value in certain regions. Blue points correspond to samples in the blue regions in Fig. 14, and green points to those in the green regions. As a reference, red points corresponding to blocks in the red region in Fig. 12 (motion-free scan) are included as well.

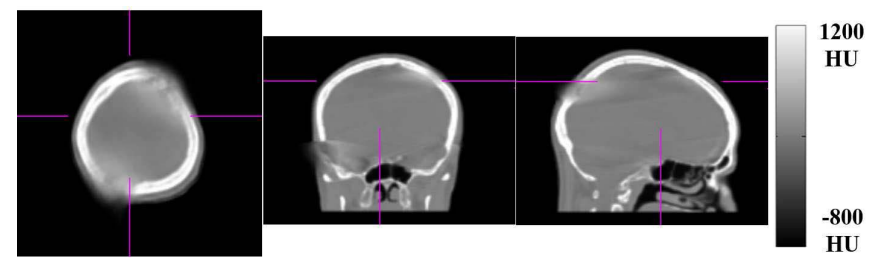

(a)

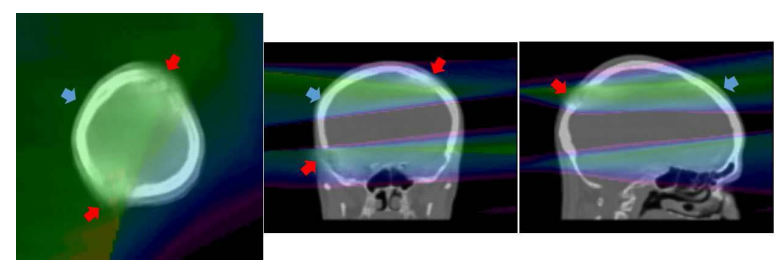

(b)

Fig. 16. Result of the simulation using the head phantom and the motion of Fig. 8. (a) MLEM reconstruction after motion correction. (b) Overlay between the Tuy map [Fig. 14(a)] and the image in (a). Red arrows indicate the distorted regions, blue arrow indicates a region which is artifact-free, although the corresponding Tuy values are relatively high. The Tuy values are all greater than 0.4 at the locations indicated by the arrows. The intensity range of the Tuy map is $[0,0.8]$.

successfully identified the regions, where data-insufficiency caused artifacts in the motion-corrected reconstruction images (red arrows). But similar to the last section, we observed that some regions of high Tuy values appear artifact-free (blue arrows). However, this does not contradict the notion that the Tuy map successfully identified the regions, where data-insufficiency might induce artifacts.

\section{E. Two Simulations Concerning Interior-Like Problem}

1) Transaxial Data Truncation From Reduced Fanbeam Angle: Fig. 19(a) shows the Tuy map for the interior-like problem created by reducing the fanangle. Near the center of the object, the truncation produced a central low Tuy value region transaxially, surrounded by high Tuy values. The high Tuy values indicate incomplete sampling due to the transaxial truncation. 


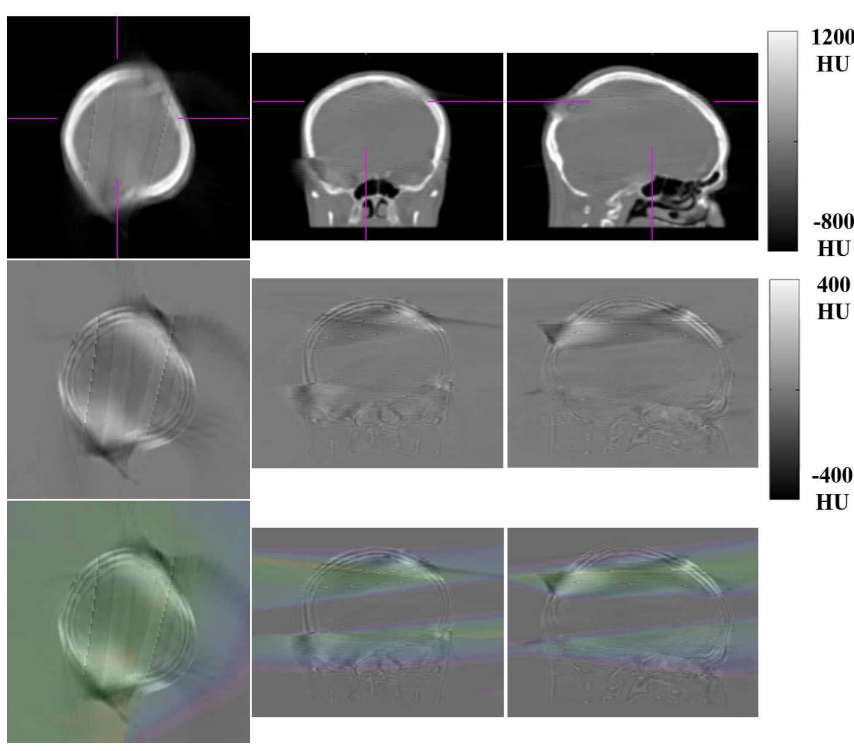

Fig. 17. Top: SART reconstruction (same planes as in Fig. 16). Middle: difference between the MLEM and SART reconstructions. Bottom: difference image with the Tuy map in overlay.

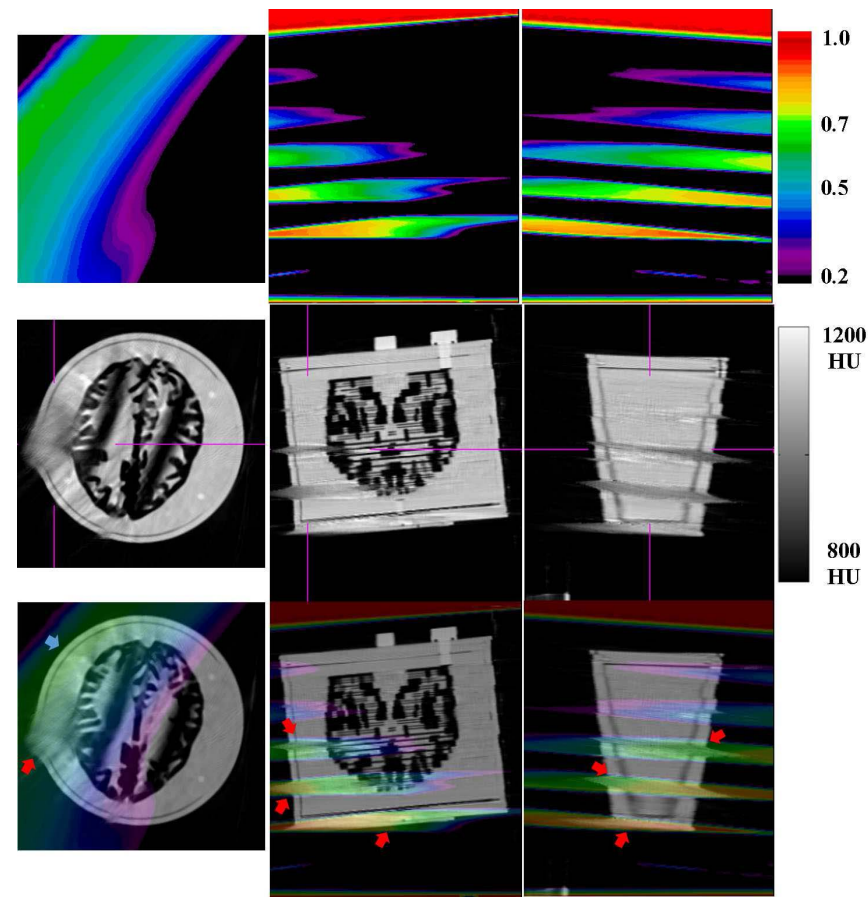

Fig. 18. Result of the phantom scan. Middle: MLEM reconstruction image (cropped to display the phantom) after motion correction using the measured motion; top: corresponding Tuy map; bottom: overlayed image. Red arrows indicate the distorted regions, blue arrow indicates a region which is artifactfree, although the corresponding Tuy values are relatively high. At all regions indicated by arrows, the Tuy values are over 0.5 .

As explained in Section II-G, it was expected that multiple solutions exist inside the interior region, even though the sampling was complete there. A first reconstruction was performed, starting from a uniform image. The reconstruction converged to an under-estimated solution in the interior region [Fig. 20(a)-left]. To check the uniqueness of this solution, we started another reconstruction from a different image, which was a reference image reconstructed from motion-free

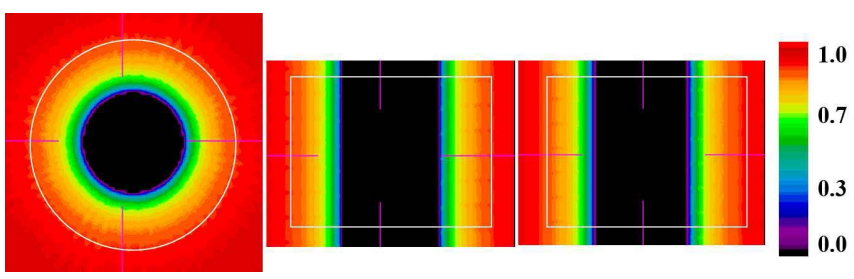

(a)

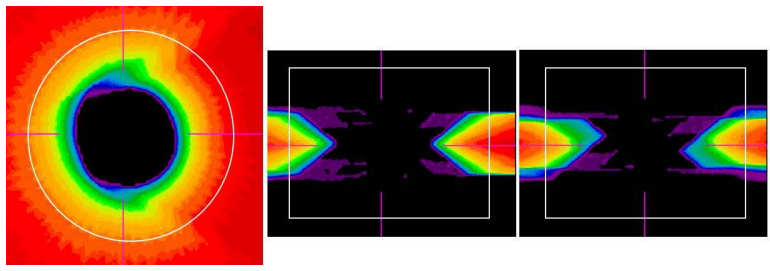

(b)

Fig. 19. From left to right: coronal, sagittal, and transaxial views of the Tuy map, with the contour (white) of the object in overlay. (a) For the interiorlike problem produced by reducing the fanangle (Section IV-E1). (b) For the problem created by the view-dependent object motion (Section IV-E2).

projections of the multisphere phantom. The reconstruction starting from the reference image converged to a solution [Fig. 20(a)-right] very close to that starting reference image. A third reconstruction was initialized with a linear combination of the two solutions. The reconstructed image (not shown here) was different from the previous two solutions and remained essentially unchanged after several iterations, confirming that the problem has multiple solutions. Consequently, the reconstruction problem does not have a unique solution in the interior region of low Tuy values. Therefore, in this interior-like problem one cannot conclude that a local low Tuy value region always indicates that region can be reconstructed exactly.

2) Data Truncation From Motion: The simulations of Section IV-E1 were repeated in the presence of the designed motion. Fig. 19(b) confirms that an interior region was created: the motion-induced sampling incompleteness yielded high Tuy values, surrounding a region, where the local Tuy value was low. Very similar results were found for all reconstructions [Fig. 20(b)], compared to ones from Section IV-E1.

\section{DISCUSSION}

Our results have verified that in the context of motioncorrected helical CT, a region of high Tuy values indicates a likelihood of artifacts there, due to data-insufficiency in agreement with and slightly generalizing the main result of [11]. With the Tuy map, one can distinguish the artifacts due to data-insufficiency from other types of artifacts, e.g., from beam hardening or motion blurring that could be corrected by using a reconstruction based on a more accurate forward model.

The Tuy map provides a general approach to assess data-insufficiency, potentially useful for many tomographic geometries like helical CT, cone-beam CT with nonstandard trajectories or SPECT with nonstandard collimators and/or trajectories, regardless of motion. The algorithm described in Section II-A can easily be modified for a specific geometry. 


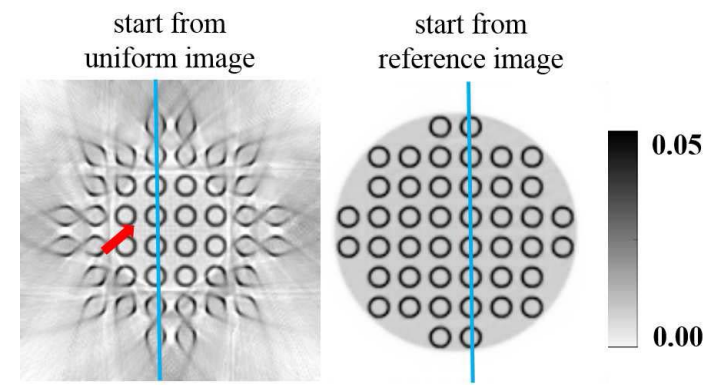

(a)

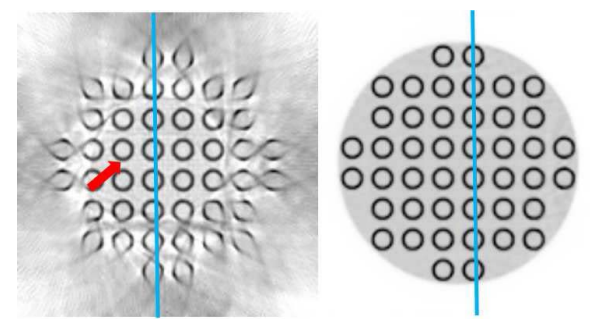

(b)

Fig. 20. Coronal views (central-slice position) of reconstruction images after 1000 updates from (a) reducing the fanangle (Section IV-E1) and (b) synthetic motion (Section IV-E2). The red arrow indicates the dc-shift in the interior region. The blue lines refer to Fig. 21.

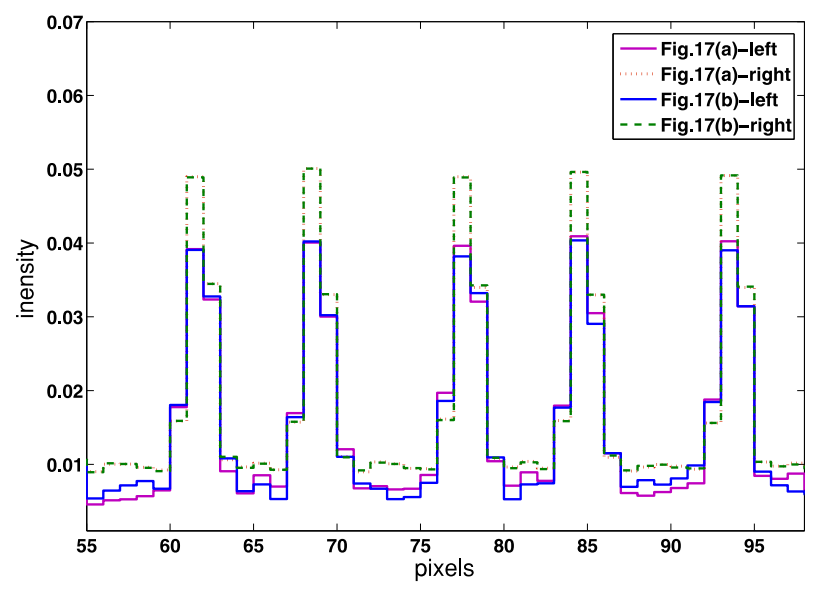

Fig. 21. Profiles along the blue lines in Fig. 20: the maximum difference between Fig. 20(a)-left and Fig. 20(a)-right is $20.6 \%$ of the maximum intensity; the maximum difference between Fig. 20(b)-left and Fig. 20(b)-right is $24.9 \%$.

One issue is that finding the complete set of measured projection lines (step 1 in Fig. 1) can be time consuming. Depending on the scan features, this step can be further optimized. For common trajectories, e.g., circle and helix, it would be possible to compute the optimal projection line analytically without searching through all available projection lines. For other trajectories, it is still very likely that the optimal projection line will occur in a short and predictable segment of the trajectory, which reduces the search space. For one particular voxel, searching can start from the index of an optimal projection line of a neighboring voxel that is already computed, which could reduce the computational burden significantly. In this paper, we applied this modification to the program in all the simulations, where no motion was present.
As shown in Figs. 12, 13, and 15, and contrary to theory, Tuy values were larger than zero in the static simulation. This is due to the discretizations described in Section II-A. Based on the multisphere phantom simulation, we consider that if the Tuy value is larger than a threshold (0.02, see Fig. 15) in a local region, some data-insufficiency exists and it can create artifacts in that region. The severity of the potential artifacts is roughly proportional to the local Tuy value.

In all the simulations there was a small intentional geometric mismatch between the geometry of the simulated data and the geometries used for calculating the Tuy map and for forward- and back-projection in the reconstructions. The purpose of this mismatch was to avoid unrealistically "perfect" data, sometimes called "the inverse crime." Otherwise, the simulation was ideal: noise, scatter, and beam hardening were not simulated, because we wanted to focus on datainsufficiency, avoiding other confounding sources of artifacts. An expectation maximization reconstruction was chosen as the reconstruction algorithm (MLEM), but the Tuy map is independent of the reconstruction method. For regions, where the data are insufficient, any reconstruction algorithm may produce a wrong solution (unless it would make use of additional prior knowledge, but this is not considered in this paper). We also tested all the simulations with another iterative reconstruction algorithm, i.e., SART (Fig. 17). All results were similar to those in Section IV.

The incompleteness in helical CT was caused by two main effects: 1) the effective trajectory and 2) truncation. The effective trajectory could be incomplete even for an ideal conebeam tomograph, where all projections would be untruncated. For example, if the object moved in the direction of the scanner axis with the same speed as the bed but in the opposite direction, then the effective trajectory would become circular, which is known to be incomplete except for the central slice. The limited axial coverage of the clinical CT system produces additional data loss, which could create data-insufficiency for an otherwise complete effective trajectory. For example, in the rolling phantom study, careful examination of the motion showed that the complex behavior of the effective trajectory in the axial direction, combined with axial truncation, generated the data-insufficiency. Fortunately, the Tuy value approach verifies any data-insufficiency, independent of its causes.

A high Tuy value in the Tuy map indicates datainsufficiency, however, the interpretation of a zero Tuy value is less obvious. Specifically, in the situation, where the scan produces regions with increased Tuy values, we cannot conclude that exact reconstruction is possible in the region of low Tuy values. In particular, for our example of the interior-like problem, we observed that the reconstruction problem does not have a unique solution inside the interior region, although the image quality inside is typically acceptable. However, if the Tuy value is close to zero everywhere, then it is believed that the data are sufficient for exact reconstruction everywhere. This notion is generally accepted although not mathematically proven [5] except in the 2-D case.

We have observed that data-insufficiency only appears to produce artifacts if the object being scanned has specific structural features at that point, in particular discontinuities that 
are parallel to missing projection lines. In contrast, no obvious artifacts were found in high Tuy value regions, where the missing projection lines were perpendicular to surfaces of discontinuities. However, this does not contradict the notion that the Tuy map provides a useful warning whenever artifacts could be present because of incomplete sampling.

The proposed Tuy value computation (Fig. 1) is similar to that of Metzler et al. [8], [12] and Liu et al. [14], but with some differences in the details. In [8], voxels were identified as either belonging or not to the fully sampled region, without identifying the degree of insufficiency. In [12] and [14] the degree of insufficiency was quantified as the fraction of projection lines that are missing, whereas in this paper, the insufficiency was quantified as the largest angle of missing lines. We have assumed that one set of missing projections covering a large angle is worse than many sets associated with small angles. The validity of this assumption remains to be investigated.

\section{CONCLUSiOn}

We have introduced a data-insufficiency measure that we call the Tuy map, the purpose of which is to quantify on a scale of zero to one, the degree of data-insufficiency at each point in the reconstructed image. Zero indicates no insufficiency, and one indicates severe insufficiency and is a signal that artifacts are likely to appear in that location. The Tuy map is calculated directly from the geometry of the acquisition: from the effective trajectory (the inverse motion applied to the physical trajectory) and the truncations of the projections. The local Tuy values are independent of the object scanned, and are independent of the choice of the algorithm subsequently used for image reconstruction. Whether or not artifacts appear, where high Tuy values occur depends on the object being imaged and not on the reconstruction algorithm (unless the algorithm is using other prior information). Low Tuy values do not guarantee accurate artifact-free reconstructions because a 3-D effect similar to the 2-D interior problem can occur.

Our studies which corroborated the above findings were all performed in the context of motion-corrected helical CT scanning, but we believe that the principles are general and can be applied to other scan situations and other modalities where, at least to first order, the imaging model involves truncated or untruncated cone-beam projections.

\section{REFERENCES}

[1] J. Nuyts, J.-H. Kim, and R. Fulton, "Iterative CT reconstruction with correction for known rigid motion," in Proc. 11th Int. Meeting Fully $3 D$ Reconstruct. Radiol. Nucl. Med., 2011, pp. 132-135.
[2] J.-H. Kim, J. Nuyts, A. Kyme, Z. Kuncic, and R. Fulton, "A rigid motion correction method for helical computed tomography (CT)," Phys. Med. Biol., vol. 60, no. 5, pp. 2047-2073, Mar. 2015.

[3] J.-H. Kim et al., "Correction for human head motion in helical X-ray CT," Phys. Med. Biol., vol. 61, no. 4, pp. 1416-1438, 2016.

[4] T. Sun, J.-H. Kim, R. Fulton, and J. Nuyts, "An iterative projectionbased motion estimation and compensation scheme for head X-ray CT," Med. Phys., vol. 43, no. 10, pp. 5705-5716, 2016.

[5] R. Clackdoyle, "Fully 3D reconstruction theory in perspective," in Proc. 8th Int. Meeting Fully 3D Reconstruction Radiol. Nucl. Med., 2005, pp. 64-69.

[6] S. S. Orlov, "Theory of three dimensional reconstruction. I. Conditions for a complete set of projections," Soviet Phys. Crystallography, vol. 20, no. 3, pp. 312-314, 1975.

[7] H. K. Tuy, "An inversion formula for cone-beam reconstruction," SIAM J. Appl. Math., vol. 43, no. 3, pp. 546-552, 1983.

[8] S. D. Metzler, J. E. Bowsher, and R. J. Jaszczak, "Geometrical similarities of the Orlov and Tuy sampling criteria and a numerical algorithm for assessing sampling completeness," IEEE Trans. Nucl. Sci., vol. 50, no. 5 , pp. $1550-1555$, Oct. 2003

[9] H. Kudo, T. Rodet, F. Noo, and M. Defrise, "Exact and approximate algorithms for helical cone-beam CT," Phys. Med. Biol., vol. 49, no. 13, pp. 2913-2931, 2004.

[10] G. Wang, Y. Ye, and H. Yu, "Approximate and exact cone-beam reconstruction with standard and non-standard spiral scanning," Phys. Med. Biol., vol. 52, no. 6, pp. R1-R13, 2007.

[11] D. V. Finch, "Cone beam reconstruction with sources on a curve," SIAM J. Appl. Math., vol. 45, no. 4, pp. 665-673, Aug. 1985.

[12] S. D. Metzler, K. L. Greer, and R. J. Jaszczak, "Helical pinhole SPECT for small-animal imaging: A method for addressing sampling completeness," IEEE Trans. Nucl. Sci., vol. 50, no. 5, pp. 1575-1583, Oct. 2003.

[13] R. Clackdoyle and F. Noo, "Cone-beam tomography from 12 pinhole vertices," in Proc. IEEE Nucl. Sci. Symp. Conf. Rec., vol. 4. San Diego, CA, USA, 2002, pp. 1874-1876.

[14] B. Liu et al., "Completeness map evaluation demonstrated with candidate next-generation cardiac CT architectures," Med. Phys., vol. 39. no. 5, pp. 2405-2416, May 2012.

[15] Y. Ye, S. Zhao, H. Yu, and G. Wang, "A general exact reconstruction for cone-beam CT via backprojection-filtration," IEEE Trans. Med. Imag., vol. 24, no. 9, pp. 1190-1198, Sep. 2005.

[16] R. Clackdoyle and M. Defrise, "Tomographic reconstruction in the 21st Century: Region-of-interest reconstruction from incomplete data," IEEE Signal Process. Mag., vol. 27, no. 4, pp. 60-80, Jun. 2010.

[17] Z. Yu et al., "Shift-invariant cone-beam reconstruction outside R-lines with a disconnected source trajectory," in Proc. IEEE Nucl. Sci. Symp. Conf. Rec., Anaheim, CA, USA, 2012, pp. 3447-3452.

[18] B. Peterson, M. Datar, M. Hall, and R. Whitaker, "GPU accelerated particle system for triangulated surface meshes," in Proc. Symp. Appl. Accelerators High Perform. Comput., 2010.

[19] L. A. Shepp and Y. Vardi, "Maximum likelihood reconstruction for emission tomography," IEEE Trans. Med. Imag., vol. 1, no. 2, pp. 113-122, Oct. 1982.

[20] H. M. Hudson and R. S. Larkin, "Accelerated image reconstruction using ordered subsets of projection data," IEEE Trans. Med. Imag., vol. 13, no. 4, pp. 601-609, Dec. 1994.

[21] B. De Man and S. Basu, "Distance-driven projection and backprojection in three dimensions," Phys. Med. Biol., vol. 49, no. 11, pp. 2463-2475, Jun. 2004.

[22] A. Andersen, "Simultaneous algebraic reconstruction technique (SART): A superior implementation of the ART algorithm," Ultrason. Imag., vol. 6, no. 1, pp. 81-94, 1984.

[23] F. Natterer, The Mathematics of Computerized Tomography. Philadelphia, PA, USA: Soc. Ind. Appl. Math., 2001, ch. 6, p. 159. 